\title{
The Morinaga misalignment effect with circular stimulus elements
}

\author{
R. H. DAY and R. T. KASPERCZYK \\ Monash University, Clayton, Australia
}

\begin{abstract}
In its original form, the Morinaga illusion is the apparent misalignment of the physically aligned apexes of oppositely facing angles. Recently, the effect has been shown to occur also with similarly arranged lines, squares, and small circles (Day, Bellamy, \& Norman, 1983). With larger circles similar in size to the angles, lines, and squares, the effect was negligible. The two experiments reported here were designed to ascertain whether the effect occurs with larger circles. The display, response mode, and method of measurement were different from those used earlier. The results indicate that the illusion, although small and relatively unstable, does occur with circles of about the same dimensions as the angles, lines, and squares of the earlier experiments.
\end{abstract}

In Figure 1, the upper and lower angles are located on one side of a vertical axis, and the central angles on the other. Although the apexes of both sets of three angles are aligned in vertical axes, they appear to be misaligned. The upper and lower apexes in each set appear to be too far inward relative to the configuration, and the central apexes too far outward. This apparent misalignment is the Morinaga illusion in its original form (Morinaga, 1941; Morinaga \& Ikeda, 1965).

The direction of apparent misalignment in Figure 1 is opposite to that expected for the Müller-Lyer illusion of extent that is also present in the figure. The horizontal extent between the upper and lower pairs of apexes appears greater than that between the central pair. This suggests that the upper and lower apexes would appear to be displaced outward rather than inward and the central apexes inward rather than outward. Because the direction of apparent displacement from alignment does not accord with expectations for the Müller-Lyer illusion, the effect has been referred to as the Morinaga paradox (Morinaga \& Ikeda, 1965; Restle, 1976). However, it is to be noted that apparent misalignment occurs independently of the Müller-Lyer illusion; the three apexes on one side of the figure exhibit the illusion in the absence of the three on the other side (Day, Bellamy, \& Norman, 1983; Restle, 1976).

In a recent investigation, Day, Bellamy, and Norman (1983) showed that the Morinaga effect also occurred when three straight lines or three squares were arranged asymmetrically on either side of an axis, as in Figure 1. With lines, the aligned end points appeared to be misaligned, and with squares, the aligned sides appeared to be misaligned. The apparent misalignment of line ends was about the same in magnitude as that of right-angle

The authors' mailing address is: Department of Psychology, Monash University, Clayton, Victoria 3168, Australia.
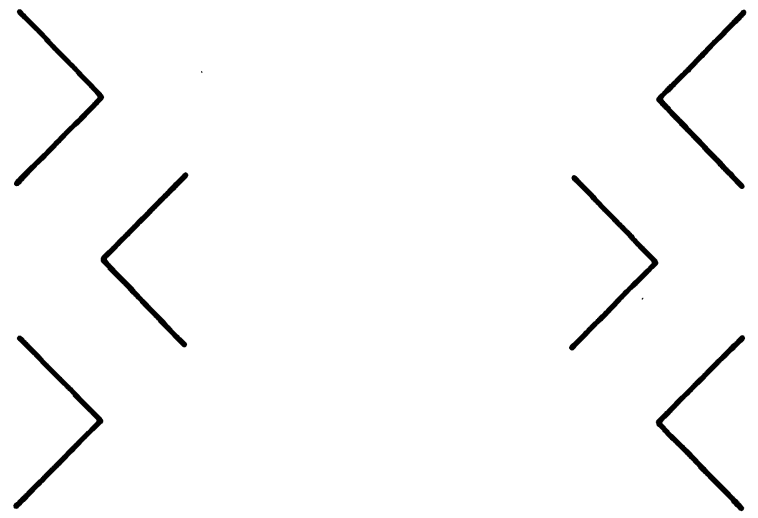

Figure 1. The Morinaga misalignment illusion. The aligned apexes of the three angles appear to be misaligned with the central apex apparently too far outward and the upper and lower apexes too far inward relative to the figure.

apexes, whereas that of the sides of squares was markedly less. The results with circles were inconsistent. With relatively small $(5-\mathrm{mm})$ circles, apparent misalignment of tangential points on the circumferences was always positive, that is, in the same direction as the displacement in Figure 1. With larger circles similar in dimensions to the lines and angles (15-20 $\mathrm{mm}$ across), apparent misalignment in three separate experiments failed to achieve statistical significance. In one experiment, the effect was slightly negative, in a second, slightly positive, and in a third, close to zero.

The reason for the absence of an illusion with the larger circles is not immediately obvious. The asymmetrical configuration formed by three circles is essentially the same as that formed by three angles, three lines, or three squares. However, it is conceivable that the task of aligning hypothetical points tangential to an axis is more difficult than that of aligning more clearly defined points such as apexes and line ends. The greater variance 
of the misalignment scores with the larger circles reported by Day, Bellamy, and Norman (1983) suggests that this might have been so. This possibility, which was not investigated in the earlier series, was the basis of the second experiment reported here.

The question concerning whether the Morinaga misalignment effect occurs with aligned points on circle circumferences is relevant to an explanation of the illusion. For example, if the effect persists with circular elements, then, given the range of elements with which it has already been shown to occur, the form of the configuration, rather than the forms of the individual elements, could reasonably be regarded as the main determinant. If, on the other hand, the illusion does not occur with circular elements, some common feature of angles, lines, and squares not shared by circles suggests itself as a primary determinant.

The two experiments described here were designed to ascertain whether the Morinaga effect occurs when the configuration is composed of three circles tangentially aligned along vertical or oblique axes. Although the figure and the general procedure were the same as earlier, the form of the display, the mode of responding, and the method of measurement were different; the figures were displayed on an oscilloscope, adjustments to apparent alignment were made by pressing keys, and the measurement of misalignment was carried out automatically. In Experiment 1, the figure comprised simply three circles (Figure 2). Since, as suggested above, the inconsistency between the outcomes of earlier experiments could have been due to the indefiniteness of the points to be aligned, these were clearly indicated in Experiment 2 by the ends of lines through the centers of the circles.

\section{GENERAL METHOD}

Since the source and number of subjects, apparatus, and procedure were the same throughout, they can conveniently be described together.

\section{Subjects}

The subjects were recruited from among undergraduates and were paid for their services. A separate group of 12 subjects participated in each experiment.

\footnotetext{
Apparatus

The stimulus figures were displayed on an $x-y$ plotter screen (Hewlett-Packard 1310A) and were generated by a microprocessor (Sierra Data Sciences SBC-100) with 12-bit D/A converters. The microprocessor was programmed to present the figures in a random order, to randomize the direction and distance of the starting points from true alignment, and to record the direction and magnitude of misalignment after each response. A black occluding mask with a circular aperture $24 \mathrm{~cm}$ in diameter was placed over the face of the plotter screen. The stimulus figures were presented about the center of the circular field. To reduce brightness to a comfortable level, a blue gelatin filter was placed behind the aperture. Thus, the figures were blue in a dark field. Their luminance was about $0.6 \mathrm{~cd} / \mathrm{m}^{2}$.

The direction of movement of the center element (see Figure 2) was controlled by two directionally marked keys on a keyboard. The direction of movement was always orthogonal to
}

the axis of physical alignment of tangent points. When the keys were held down, the movement of the center element was continuous, and when they were tapped, it was stepwise, moving $0.15 \mathrm{~mm}$ for each tap. A third key, when tapped by the subject, brought up the next figure in the stimulus series.

The viewing distance was about $114 \mathrm{~cm}$; at $1 \mathrm{~cm}$, the display subtended a visual angle of $.5 \mathrm{deg}$. Head position and movements were not restrained. Low room illumination was provided by a reading lamp in a corner of the laboratory behind the subject.

\section{Procedure}

The subject's task throughout was to adjust the position of the central circle so that the unmarked (Experiment 1) or marked (Experiment 2) point on the right of the circumference appeared to be perfectly aligned with similar points on the left of the circumferences of the two outer circles. Continuous or discontinuous adjustment movements were allowed. An experimental session consisted of instructions, 4 practice trials, and 12 experimental trials (4 per condition). During instruction, the subjects were shown the display, and the use of the keys was demonstrated. Questions were encouraged and answered. A trial consisted of movement of the center circle from a position of obvious misalignment to one of apparent alignment of the three tangent points on the circumferences. A trial began with the center circle on either side of the axis of physical alignment. There were four trials with each figure, two from starting positions on one side of the axis and two from positions on the other side. There were 4 practice trials and 12 experimental trials, 4 of the latter for each of the three orientations of the figure shown in Figure 2. The order of presentation of the figure arrangements and the starting positions were differently randomized for each subject. The score throughout was the difference between the true and the apparent axis of alignment (in millimeters). Misalignment scores that compensated for the illusion were treated as positive, and those in the opposite direction, as negative.

\section{EXPERIMENT 1}

Experiment 1 was essentially a repetition of earlier experiments with circular elements (Day, Bellamy, \& Norman, 1983). The main differences were the computercontrolled display, keypress response system, and automatic scoring. The stimulus figures were presented with the alignment axes vertical and oblique (Figure 2), since the earlier experiments had consistently shown that the illusion was greater with the oblique arrangement, presumably an instance of the oblique effect (Appelle, 1972). It was of theoretical interest to establish whether the difference in magnitude of illusion between the two axes obtained for circles.

\section{Method}

Stimulus figures. The three circular elements arranged vertically and in the two 45-deg oblique directions are shown in Figure 2. The circles were $15 \mathrm{~mm}$ in outer diameter and were separated by $25 \mathrm{~mm}$ between adjacent tangential points of physical alignment. Following the earlier observations with angle and line elements (Day, Bellamy, \& Norman, 1983) it was expected that, if apparent misalignment occurred with circular elements, it would be greater for the two oblique orientations of the figure than for the vertical orientation.

\section{Results}

The means and standard deviations of the misalignment scores are also shown in Figure 2. It can be seen 


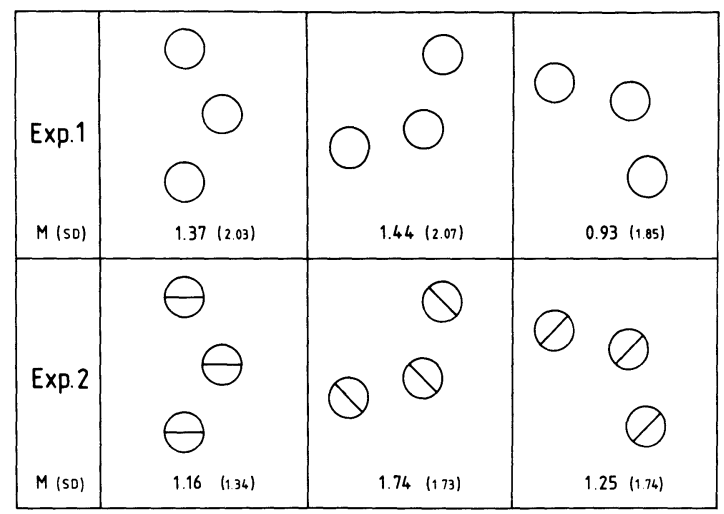

Figure 2. The figures for Experiments 1 and 2, with the mean misalignment scores in millimeters. Standard deviations are shown in parentheses.

that all means are positive. However, it is to be noted that the scores for between about 1.2 and $1.7 \mathrm{~mm}$ are generally smaller than those for between about 1.6 and $2.7 \mathrm{~mm}$ found earlier for angles and lines of similar dimensions. Inspection of the data shows that three negative scores occur for the right oblique arrangements, four for the left oblique, and four for the vertical. It can be seen, too, that the means for the two oblique arrangements are not notably different from those for the vertical.

A one-way analysis of variance showed that the differences between the three means are not significant $[F(2,22)=1.45, p>.05]$. Separate $t$ tests on the scores for each condition showed that, whereas the means for the vertical and right oblique arrangements are different from zero $\left[t_{v}(11)=2.35, p<.05 ; t_{r . o b l .}(11)=2.41\right.$, $\mathrm{p}<.05]$, that for the left oblique arrangement is not [ $\mathrm{t}_{\text {l.obl. }}(11)=1.75, \mathrm{p}>.05$ ]

In summary, whereas the misalignment effect overall was weak relative to that for angle and line figures, it was significant for two of the three circle figures. Contrary to earlier observations, there was no difference between the size of the effect for vertical and oblique arrangements of the three elements. These outcomes are discussed below.

\section{EXPERIMENT 2}

Unlike the apexes of angles and the ends of lines, tangential points on circumferences are perceptually indefinite. It is possible that the absence of a significant effect in one of the three conditions in Experiment 1 and the generally weak effect for the other two were due to difficulty in identifying the points on the circumferences to be aligned. Such difficulty could be expected to result in variable direction of adjustment to apparent alignment. Perceptual difficulty in identifying the points of alignment would also explain the difference in the effect for large and small circles: The smaller the circle, the easier, presumably, it would be to locate the point.

This interpretation of the weaker and less stable Morinaga effect with circular elements was tested in Experiment 2 . The points to be aligned were clearly marked by the ends of lines drawn through the centers of the circles orthogonal to the alignment axis. It could be reasonably presumed that the difficulty of identifying the alignment points would be no greater than that with line elements.

\section{Method}

Stimulus figures. The three figures are shown in Figure 2. They were the same in dimensions and orientation as those in Experiment 1. The only difference was the addition of diameter lines. The right ends of the lines through the outer circles and the left end of that through the central circle marked the points to be aligned.

\section{Results}

The mean misalignment scores and their standard deviations are shown in Figure 2. Although the three means are of about the same size as those for Experiment 1 , the standard deviations are slightly smaller. Inspection of individual scores shows that there were two negative scores for the vertical arrangement, two for the right oblique arrangement, and three for the left oblique arrangement. A one-way analysis of variance indicated that there is no difference between the three means $[F(2,22)=.87]$. However, separate $t$ tests showed that all three means are significantly different from zero $\left[\mathrm{t}_{\mathrm{v}}(11)=3.02, \mathrm{p}<.02 ; \mathrm{t}_{\mathrm{r} . \text { obl. }}(11)=3.48, \mathrm{p}<\right.$ $\left..01 ; t_{1 . o b l .}(11)=2.48, p<.05\right]$. It can be noted that, in general, the levels of significance of the means relative to zero effect are greater than those in Experiment 1.

\section{DISCUSSION}

In Experiment 1, the Morinaga misalignment effect occurred under two of the three conditions of orientation, and in Experiment 2, under all three. On the basis of these outcomes, it is reasonable to conclude that the effect does occur when the stimulus figure is made up of circles of about the same dimensions as angles, lines, and squares that have been shown to give rise to consistent effects. However, it is to be noted that, as well as an insignificant mean in one of the oblique orientation conditions in Experiment 1, negative scores occurred in all three conditions in both experiments. Furthermore, the means were smaller than those reported earlier (Day, Bellamy, \& Norman, 1983) for lines and angles. Therefore, it must also be concluded that, although the Morinaga effect occurs with relatively large circles, it is weaker than that associated with angle and line elements.

In Experiment 2, in which diameter lines at right angles to the alignment axis of the circles were added, all three means were significant, and the standard deviations were consistently smaller than those in Experiment 1 for circles without the lines. This outcome suggests that the greater variance and instability of the effect in Experiment 1 and in the earlier experiments was due in some part to difficulties experienced by subjects in identifying the points on the circumferences to be aligned. 
It will be recalled that, in the earlier experiments, the Morinaga effect was negligible. The question can be asked as to why the outcomes of the two groups of experiments were inconsistent. Although any answer to this question is bound to be speculative, it is reasonable to assume that the light-on-dark display of the present series, the keypressing mode of responding, and the automatic measurement of misalignment contributed to the difference.

In the earlier series involving various forms of stimulus elements, the misalignment effect was invariably greater when the axis of physical alignment was oblique. Indeed, this was among the most consistent outcomes of the series. In the two experiments reported here, there was no difference in the effect between the vertical and the oblique arrangements of the elements. No explanation can be offered at this stage for the absence of a difference in the misalignment effect between the vertical and the oblique orientations for circles.

It is concluded that, under the conditions described here, the Morinaga misalignment effect, although weak relative to that with angle, line, and small circle elements, does occur with stimulus figures consisting of relatively large circles. It is also concluded that, although difficulty in identifying the points to be aligned on the circumferences contributes to the weakness of the misalignment effect with larger circles, this is not the only factor responsible.

\section{REFERENCES}

Appelle, S. (1972). Perception and discrimination as a function of stimulus orientation: The "oblique effect" in man and animals. Psychological Bulletin, 78, 266-278.

Day, R. H., Bellamy, S., \& Norman, A. (1983). On the Morinaga misalignment illusion. Journal of Experimental Psychology: Human Perception and Performance, 9, 113-125.

MorinagA, S. (1941). Some considerations on the explanations of the Mueller-Lyer illusion. Japanese Journal of Psychology, 16, 26-39.

Morinaga, S., \& Ikeda, H. (1965). Paradox in displacement in geometrical illusion and the problem of dimensions: A contribution to the study of perception. Japanese Journal of Psychology, 36, 231-238.

Restle, F. (1976). Morinaga's paradox and figure-ground orientation. Perception \& Psychophysics, 20, 153-156.

(Manuscript received for publication January 3, 1984.) 